Title : will be set by the publisher

Editors : will be set by the publisher

EAS Publications Series, Vol.?, 2003

\title{
GENIE: A GROUND-BASED PRECURSOR FOR THE IRSI-DARWIN MISSION
}

\author{
O. $\mathrm{Absil}^{1}$
}

\begin{abstract}
A definition study for a Ground-based European Nulling Interferometry Experiment (GENIE) has been initiated by the European Space Agency (ESA) and the European Southern Observatory (ESO). This instrument is planned to be installed in 2006 on the VLTI at Mount Paranal, Chile. The primary objective of the GENIE experiment is to gain experience in the design, manufacture and operation of a nulling interferometer using a Darwin representative technology. Besides its technological goal, GENIE is designed to survey exozodiacal dust clouds around nearby stars and to study the brightest of them by means of low-resolution spectroscopy. GENIE also aims at low-mass companion detection, including hot Jupiters. In this paper, the expected performances of the GENIE interferometer are investigated as a function of the observation wavelength and of the performances of critical subsystems.
\end{abstract}

\section{Introduction}

The principle of nulling interferometry was proposed by R. Bracewell in 1978. The purpose of this technique is to achieve a destructive interference for an on-axis target while letting through the signal from a nearby companion or a surrounding dust cloud. This can be achieved by introducing an achromatic $\pi$ phase shift in one arm of the interferometer. Nulling interferometry has been identified as one of the most efficient ways to directly detect the light coming from an extra-solar planet, and has been selected as a baseline for ESA's Darwin mission.

In the context of the Technical Research Program leading to ESA's IRSIDarwin, a major issue is the development of a ground-based nulling interferometer to test the IRSI-Darwin technologies in an appropriate environment. The Very Large Telescope Interferometer (VLTI) at ESO (Paranal, Chile) will provide the infrastructure for the Darwin-GENIE experiment. This nulling interferometer will

\footnotetext{
${ }^{1}$ IAGL, University of Liège, B5C, 17 Allée du 6 Août, B-4000 Sart-Tilman, Belgium
} 
combine two or more of the VLTI telescopes, using all optical functions foreseen for the future Darwin Infrared Space Interferometer. The overall performance of the instrument will heavily depend on the performance of all VLTI subsystems and in particular on the adaptive optics and co-phasing subsystems.

The success of future space missions dedicated to Earth-like planet detection, such as Darwin and TPF, critically depends on the amount of exozodiacal dust around their target stars. In order to produce significant information for these space missions, GENIE must be sensitive to dust clouds about ten times as dense as our zodiacal cloud ("10-zodi cloud"). Such a dust cloud, surrounding a Sun-like star located at 10 parsecs, will be our reference target in this paper. The contrast between the star and the cloud is presented in table 1 .

Table 1. Approximate exozodiacal flux and star/cloud contrast in the case of a 10-zodi cloud around a Sun-like star at 10 pc (reference target).

\begin{tabular}{|l|c|c|c|c|}
\hline Spectral band & $\mathrm{K}$ & $\mathrm{L}$ & $\mathrm{M}$ & $\mathrm{N}$ \\
\hline Exozodiacal flux (mJy) & 0.2 & 0.5 & 0.7 & 1.1 \\
Corresponding magnitude & 16.2 & 14.2 & 13.4 & 11.3 \\
Star/cloud contrast & $1.5 \times 10^{5}$ & $3 \times 10^{4}$ & $1.4 \times 10^{4}$ & $2.5 \times 10^{3}$ \\
\hline
\end{tabular}

\section{Nulling configurations at the VLTI}

Thanks to its four 8-m Unit Telescopes (UT) and its three 1.8-m Auxiliary Telescopes (AT) which move on tracks (a fourth AT will probably be built), the VLTI offers a great number of potential nulling configurations (Fig. 1). Linear arrays are generally preferred, because their projection on the plane of the sky remains homothetic during the night, while 2-D arrays are deformed by Earth rotation.

In order to detect exozodiacal dust around nearby stars, simple nulling configurations like a two-telescope Bracewell interferometer formed of two UTs can be used. Because a nulling interferometer uses coaxial beam-combination, no image is formed: the total flux transmitted by the interference pattern (also called transmission map) is integrated on a single pixel detector. Besides the contribution from the exozodiacal cloud, the output signal of the interferometer contains contributions from the central star, from the thermal background and from potential planets orbiting the target star. Unless one of these planets is a hot giant planet, their thermal emission will be negligible compared to the infrared flux from the exozodiacal cloud. The central transmission of the Bracewell interferometer, proportional to $\theta^{2}$ along the interferometer baseline (Fig. 1), is not very deep. Therefore, the shortest baseline - $46 \mathrm{~m}$ between UT2 and 3-is recommended to produce a wide fringe pattern and thus to cancel the starlight as much as possible.

Other linear configurations are not possible with the UTs. However, three or even four aligned AT stations can easily be found (see Fig. 1). It is therefore 

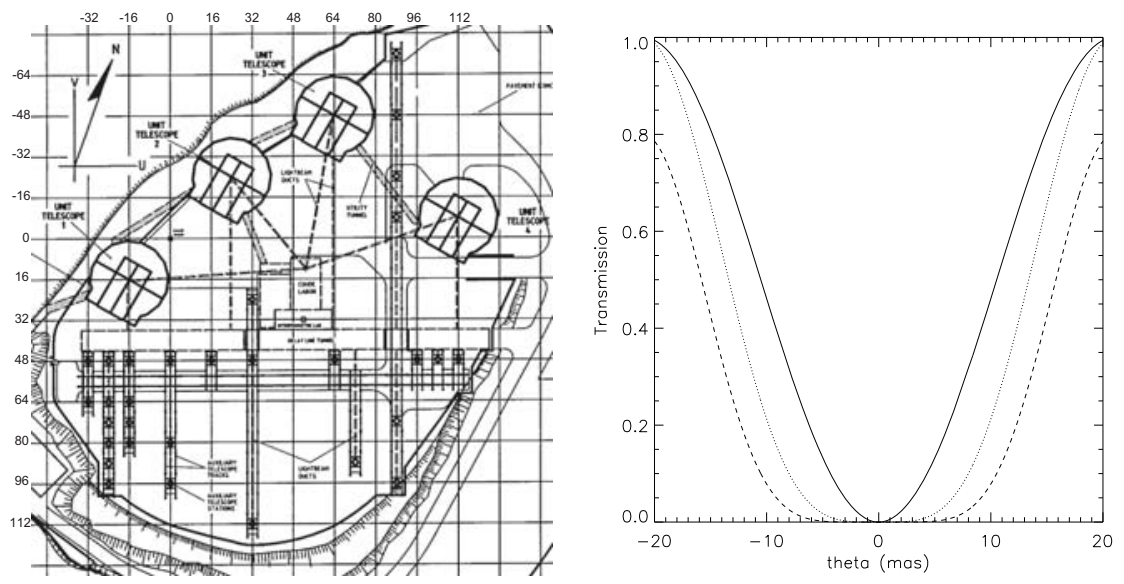

Fig. 1. Left: Map of the VLTI site, with the four Unit Telescopes and the stations for the Auxiliary Telescopes (filled circles). There are many ways to find three (or four) aligned AT stations. Right: Transmission along the baseline for a Bracewell interferometer $\left(\theta^{2}\right.$ transmission, solid line), a Degenerate Angel Cross ( $\theta^{4}$ transmission, dotted line) and an OASES interferometer $\left(\theta^{6}\right.$ transmission, dashed line).

possible to use nulling configurations with a deeper null, like the DAC and OASES configurations. Their central transmission scales respectively as $\theta^{4}$ and $\theta^{6}$. These configurations produce a lower stellar leakage, which could be a serious advantage if the calibration of the stellar signal turns out to be a limiting factor. The main drawbacks of these configurations are the complexity of their recombination scheme and their low sensitivity due to the use of the 1.8-m ATs.

In the case of exoplanet detection, the light coming from the exozodiacal cloud is an additional source of noise, and should be removed for a secure detection. A special modulation technique, called internal modulation, has been designed by Mennesson and Léger (2002) for this purpose. This technique has the advantage to remove all spurious signals, such as the background, mean stellar leakage and exozodiacal light. Internal modulation could be tested at the VLTI in a "double Bracewell" configuration, using the four UTs at the same time.

\section{Main noise sources}

The noises generated by the stellar leakage and the thermal background can both be divided into two parts: the shot noise associated to their mean value, and the noise associated to their fluctuations. For the stellar leakage, the fluctuations are due to two main effects: differential optical path delay and intensity mismatches between the arms of the interferometer. For the thermal background, the fluctuations are mainly due to temperature and emissivity fluctuations in the atmosphere and in the optical train. In this section, we investigate the sources of "fluctuation 
noises" in the case of Bracewell interferometer. These considerations can easily be generalized for a nulling interferometer formed of more than two apertures.

\subsection{Atmospheric piston}

A major source of stellar leakage fluctuations is the time-varying optical path delay (OPD hereafter) between the two arms of the interferometer: the non-homogeneity of the atmospheric refraction index induces a differential delay between the two incoming wavefronts referred to as "piston" (Fig. 2). The piston is highly variable with time, with a zero mean and a typical standard deviation of $20 \mu \mathrm{m}$ RMS for a $46 \mathrm{~m}$ baseline. The effect of differential OPD is to make the fringes move around their nominal position (Fig. 2). In the case of GENIE, this means that the central dark fringe is not centered on the stellar disk any more, so that the starlight transmission increases. In the $\mathrm{N}$ band, an OPD variation of $5 \mu \mathrm{m}$ is sufficient to get a constructive interference instead of a destructive one, and therefore, the differential OPD must be reduced by means of a fringe sensing unit (FSU). The nulling process needs a very high stability in the position of the fringes, particularly at short wavelengths. Fringe stabilization will be achieved by the PRIMA-FSU facility at the VLTI. The residual differential OPD at the output of the FSU should be about $70 \mathrm{~nm}$ RMS. This residual OPD could be further reduced by using of fringe tracker optimized for bright targets, which is not the case for PRIMA.
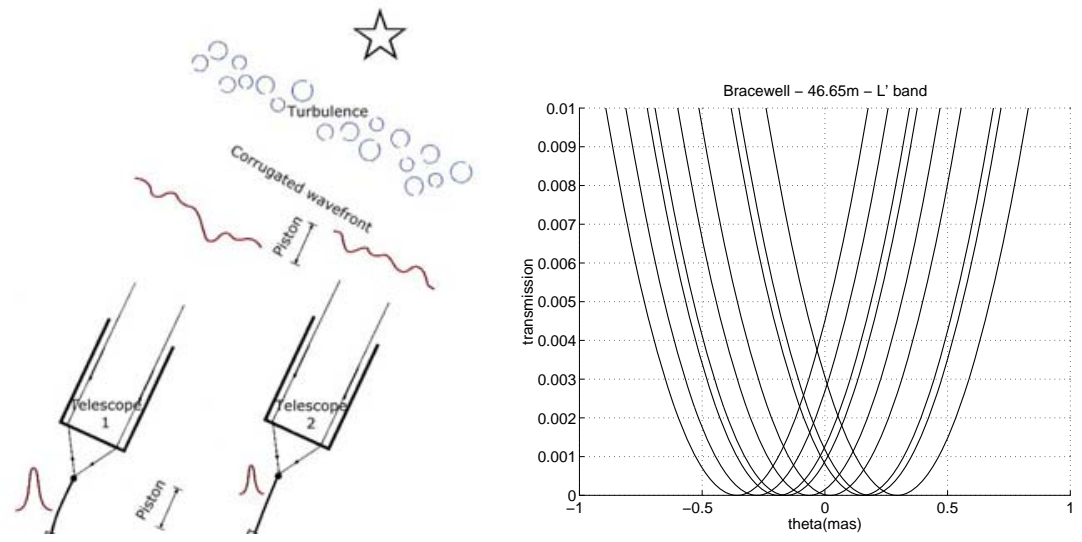

Fig. 2. Left: Differential OPD (piston) between the two arms of a fibre-linked interferometer (courtesy of G. Perrin). Right: : The central fringe moves around the centre of the field-of-view as a result of OPD fluctuations. The stellar disk is about 0.5 mas in radius in the case of a Sun-like star located at $10 \mathrm{pc}$.

The fringe jitter induced by the residual OPD has two consequences: it decreases the final cloud/star ratio by increasing the mean stellar transmission, and it introduces noise associated with the stellar leakage fluctuations. 


\subsection{Intensity mismatch}

Before recombination, the beams coming from the telescopes will be spatially filtered by single-mode fibres. The coupling efficiency into the fibres mainly depends on two parameters: the Strehl ratio delivered by the adaptive optics system and the pointing errors. If the stellar signal is not balanced in the two arms of the interferometer, the on-axis extinction will not be perfect as shown by Fig. 3. The coupling efficiencies are fluctuating with time, leading to the same consequences as the piston: an increase of the mean stellar leakage and stochastic fluctuations of the instantaneous leakage.
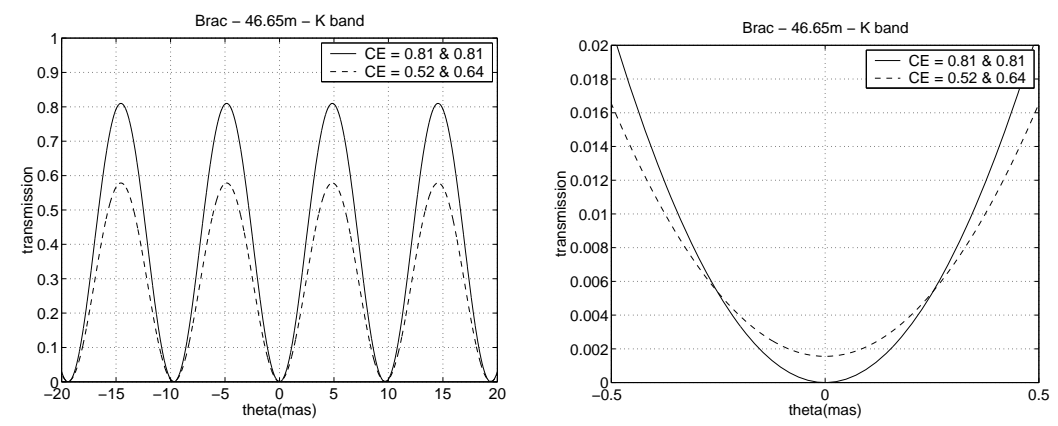

Fig. 3. Deformation of the fringe pattern due to unmatched coupling efficiencies in the two arms of a Bracewell interferometer. The maximum coupling efficiency is of $81 \%$, while the expected coupling efficiency in the $\mathrm{K}$ band is of $58 \%$ with an RMS of $12 \%$ (B. Koehler, personal communication). The left-hand figure shows that the total transmission is reduced due to the mean coupling efficiency of $58 \%$, while the right-hand figure shows the vertical deformation of the central fringe.

In order to reduce the intensity fluctuations in the arms of the interferometer, photometric signals will be extracted from the filtered beams and fed back to a control loop to equalize the stellar signal in the two beams. As in the case of OPD control, the accuracy of the intensity control is particularly crucial at short wavelengths, where the fluctuations of the coupling efficiency are larger.

\subsection{Thermal background}

An important source of noise for any ground-based infrared instrument is the thermal emission from the sky and from the telescope itself. The atmospheric background has been measured in several astronomical sites. The values in table 2 are adapted from recent observations of Cuby et al. (2000) at Paranal. The thermal emission from the warm optical surfaces depends on their emissivity and temperature. The mean temperature in the VLTI interferometric lab and delay line tunnel is about $288 \mathrm{~K}$ and the emissivity of the optical train is about $60 \%$.

In addition to its shot noise, the background contributes to the total noise by 
Table 2. Estimate integration time for 10-zodi cloud detection, taking into account only the photon noise from the thermal background and from the dust cloud itself. An overall instrumental transmission of $1 \%$ is assumed (VLTI+GENIE).

\begin{tabular}{|l|c|c|c|c|}
\hline Spectral band & K & L' & M & N \\
\hline Exozodiacal flux (mJy) & 0.2 & 0.5 & 0.7 & 1.1 \\
Mean sky brightness (Jy/ $\left.\mathrm{as}^{2}\right)$ & 0.004 & 3 & 30 & 400 \\
Blackbody at 288 K (Jy/as $\left.{ }^{2}\right)$ & 0.012 & 32 & 251 & 6567 \\
\hline UT - Background/exozodi & 0.7 & $2 \times 10^{3}$ & $2 \times 10^{4}$ & $1 \times 10^{6}$ \\
UT - Integration time (sec) & $3 \times 10^{-4}$ & 0.3 & 2 & 285 \\
\hline AT - Background/exozodi & 13 & $3 \times 10^{4}$ & $4 \times 10^{5}$ & $2 \times 10^{7}$ \\
AT - Integration time (sec) & 0.1 & 100 & 700 & 100000 \\
\hline
\end{tabular}

its fluctuations. Chopping is a technique designed to suppress the background emission and its low-frequency fluctuations by alternately measuring the flux from the scientific source and from a nearby empty sky region in order to sample the background emission. In fact, little is known about the stability of the background, particularly in the mid-infrared. In the following simulations, we will always assume that the chopping is efficient enough to remove the background fluctuations. This is a crucial requirement for N-band observations, where the background is much larger than the scientific signal.

Two chopping methods are considered for the GENIE instrument. The first one is the classical method where the secondary mirror of the telescope is repeatedly tilted towards an empty sky region to measure the background flux. Since the background is not expected to be spatially uniform, a second series of chopped measurements must be carried out with a slightly depointed primary mirror to remove the spatial gradient of background emission. Since the fringes are lost at each chopping period, the overall efficiency of this method critically depends on the time needed by the control subsystems to close their loops. Interferometry allows for another type of chopping, called "internal chopping" by opposition to the classical "external" chopping. This method, proposed by Kuchner and Serabyn (2002), requires the pupils of the telescopes to be divided into two parts to form a pair of nullers between which a phase modulation can be performed. With this chopping method, the background flux can be continuously monitored without fringe loss.

\section{Expected performances}

Prospective performances of the GENIE instrument have been computed, taking into account the noise sources discussed above, as well as the read-out noise of current infrared detectors. The noise contributions are presented in Fig. 4 in the case of L' and $\mathrm{N}$ band observations. As expected, the background is the dominant source of noise in the $\mathrm{N}$ band, where the detector noise has also an important 
contribution. In the L' band, the "fluctuation noise" due to stellar leakage becomes the main contributor. With a second stage of OPD control optimized for bright targets, the fluctuation noise would be smaller than the background noise, even in the L' band, provided that the residual OPD is smaller than $25 \mathrm{~nm}$ RMS.
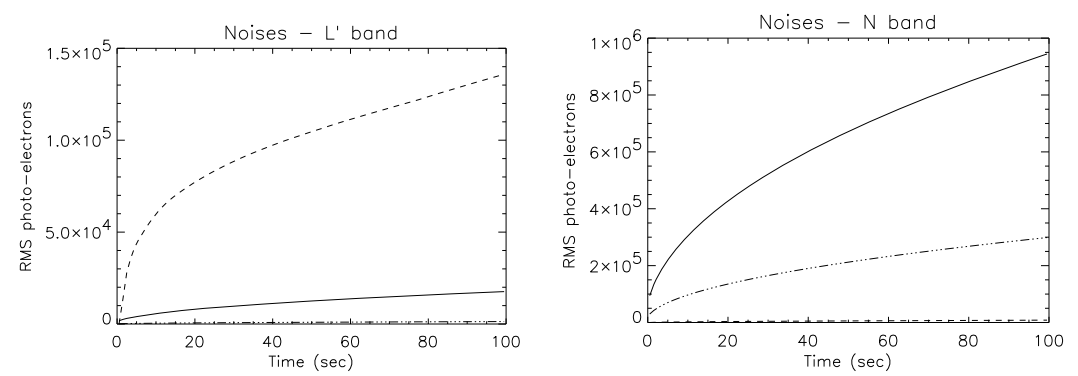

Fig. 4. Noise contributions for a UT2-UT3 Bracewell interferometer pointing at zenith towards a Sun-like star at 10 pc surrounded by a 10-zodi cloud: noise from stellar leakage fluctuations (dashed), photon noise from the mean background, mean leakage and exozodiacal cloud (solid) and detector noise (dashed-dotted).

The expected performances of the Bracewell interferometer are summarised in table 3. They prove that the detection of 10-zodi clouds around nearby stars is a realistic goal, within less than one hour of integration time. Depending on the chosen waveband, different kinds of problems will be faced: background emission at long wavelengths ( $\mathrm{N}$ band) and stellar leakage at short wavelengths ( $\mathrm{L}$ ' band). The first problem cannot be solved, unless bigger telescopes are available, while the second problem can be tackled by using other types of configurations. The DAC and OASES configurations, which have a deeper null, would reduce the contribution of the stellar leaks by a factor of about 10. However, the DAC and OASES configurations require ATs to be used. As proven in table 2, the resulting integration times would be too long in the $\mathrm{N}$ band. On the other hand, $\mathrm{K}$ and $\mathrm{L}$ ' band observations would benefit from these configurations.

In the case of hot Jupiter detection, the signal-to-noise ratio depends on the chosen target. For the brightest exoplanets (tau Boo b, 51 Peg b), we can expect the signal-to-noise ratios to be somewhat larger than those in table 3 , especially in the near-infrared where a $100 \mathrm{~m}$ baseline is sufficient to resolve the star-planet system. The $\mathrm{N}$ band is not really appropriate for hot Jupiter detection at the VLTI, because the typical angular distance between the star and the planet is of 5 mas, while the angular resolution is limited to 20 mas in the $\mathrm{N}$ band.

\section{Conclusion}

The choice of the waveband for GENIE is a crucial issue. The $\mathrm{N}$ band has two important advantages: first, it corresponds to the Darwin waveband, so that any 
Table 3. Prospective performances of a UT2-UT3 Bracewell interferometer: signals (phel) and noises (ph-el rms) in 100 seconds for a 10-zodi cloud around a Sun at 10 pc.

\begin{tabular}{|l|c|c|}
\hline Spectral band & $\mathrm{L}^{\prime}$ & $\mathrm{N}$ \\
\hline Exozodi signal & $8.3 \times 10^{4}$ & $3.0 \times 10^{5}$ \\
Star transmission & $3.0 \times 10^{-3}$ & $5.1 \times 10^{-4}$ \\
Star signal & $1.9 \times 10^{7}$ & $1.3 \times 10^{6}$ \\
Background signal & $2.9 \times 10^{8}$ & $8.9 \times 10^{11}$ \\
\hline Photon noise & $1.8 \times 10^{4}$ & $9.4 \times 10^{5}$ \\
Fluctuation noise & $1.3 \times 10^{5}$ & $9.3 \times 10^{3}$ \\
Detector noise & $1.3 \times 10^{3}$ & $3.0 \times 10^{5}$ \\
\hline SNR & 0.6 & 0.3 \\
\hline
\end{tabular}

technological development would be beneficial to the space mission, and second, it is well suited to the study of circumstellar dust. The L' band would probably be more representative of the Darwin environment, where the background and the stellar leaks have almost the same level. The L' band would also allow the use of ATs and possibly of an integrated optics beam-combiner.

The choice of the aperture configuration is probably more straightforward: the Bracewell configuration is recommended because of its simplicity. Due to the increased complexity of their recombination scheme, the DAC and OASES interferometers would only be recommended if the stellar leakage calibration turns out to be a major issue. Once again, in order to reduce the complexity, external chopping is recommended, provided that it can achieve the required accuracy of background subtraction.

Finally, testing internal modulation with the double Bracewell configuration would be a major step towards Darwin, especially for data reduction in a Darwinlike environment.

\section{Acknowledgments}

This Research was supported through a European Community Marie Curie Fellowship. The author is solely responsible for the information communicated, published or disseminated. It does not represent the opinion of the Community. The Community is not responsible for any use that might be made of data appearing therein.

\section{References}

Bracewell, R. 1978, Nature, 274, 780.

Cuby, J., Lidman, C. and Moutou, C. 2000, The Messenger, 101, 2.

Kuchner, M. and Serabyn, G. 2002, accepted for publication in ApJ.

Mennesson, B. and Léger, A. 2002, submitted to Icarus. 